\section{Jumper's knee}

\section{R V P de Villiers MMed (RadD) SA}

Department of Radiology, Tygerberg Academic Hospital and University of Stellenbosch. Tygerberg, W Cape

\section{Abstract}

Patellar tendinopathy or 'jumper's knee' is a common cause of anterior knee pain in athletes. This condition is often resistant to therapy and can cause a premature end to a professional sporting career. MRI and highdefinition ultrasound are the modalities of choice for evaluating the athlete with anterior knee pain. Ultrasound with a linear, high-resolution 10 or 12 $\mathrm{MHz}$ probe is suggested. Comparison is always made with the presumed normal contralateral tendon. MRI, although more expensive, is not operator-dependent. It also demonstrates a more global picture. Fluid-sensitive studies are used in the axial and sagittal planes. The pathology of patellar tendinopathy represents a tendinosis, rather than a tendonitis, due to the absence of inflammatory cells.

\section{Introduction}

Athletes in jumping sports such as basketball, high jump, pole vault, volleyball and netball often suffer from anterior knee pain caused by patellar tendinopathy or 'jumper's knee'. This condition is often resistant to therapy and can cause a premature end to an elite or professional sporting career.
Clinically these athletes present to the physiotherapist or sports physician with pain just below the patella that is aggravated by jumping. Pain is aggravated by stressing the extensor mechanism, e.g. with weight training or squatting. On examination the patient is tender at the junction between the patella and the patellar tendon.

\section{Imaging approach}

MRI and high-definition ultrasound are the modalities of choice for the jumping athlete with anterior knee pain. ${ }^{2-1}$

Plain radiography is almost always negative, unless a stress fracture of the inferior patellar pole is present., ${ }^{2,3}$

Ultrasound with a linear, high-resolution 10 or $12 \mathrm{MHz}$ probe shows loss of the normal fibrillar echogenicity of the infrapatellar tendon. Comparison is always made with the presumed normal contralateral tendon. Technically the probe must always be directed perpendicular to the tendon to avoid artifactual anisotropism. The changes may vary from grade 1 tendinosis with gener-

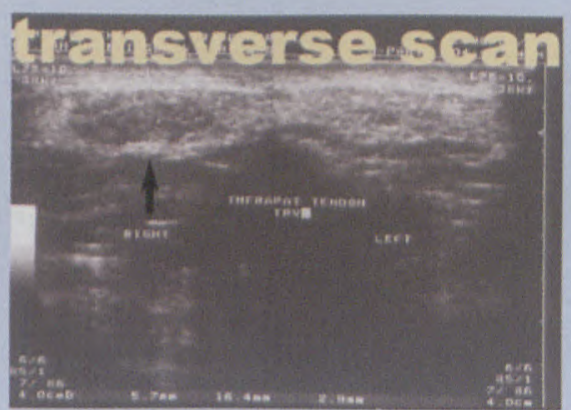

Fig. 1. Transverse ultrasound scan of infrapatellar tendon. Right tendon shows hypoechoic tendinopathic changes.

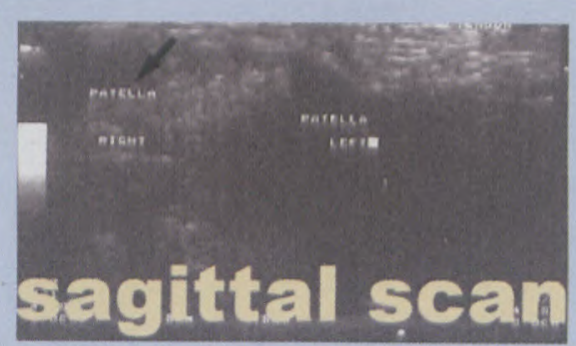

Fig. 2. Sagittal ultrasound scan shows hypo echoic tendinopathic changes of the posterior superior aspect of the right infrapatellar tendon. The left infrapatellar tendon is normal and shows the normal fibrillar pattern.

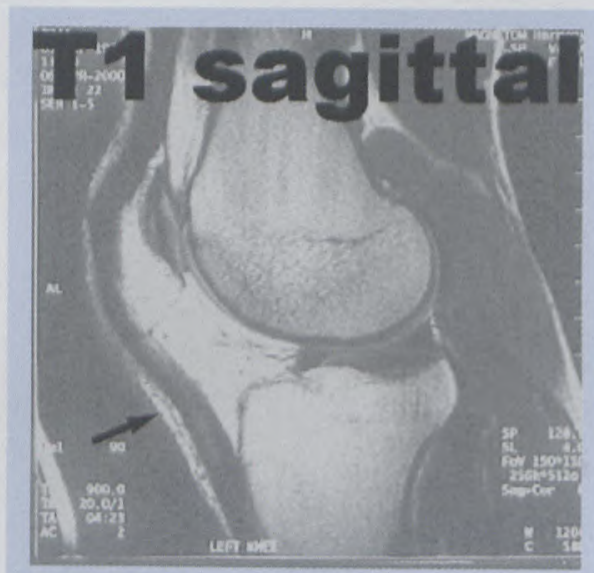

Fig. 3. T1 sagittal MRI shows subtle hyperintense changes in the whole infrapatellar tendon. Compare the signal in the suprapatellar tendon with the signal in the infrapatellar tendon. Note the fusiform swelling in the tendon.

alised hypoechoic tendon swelling to focal hypoechoic areas. Classically, the posterior tendon fibres are predominantly involved. Inflammatory changes in the infrapatellar or Hoffa's fat pad may be noted. This is demonstrated as hypoechoic shadowing compared with the contralateral fat pad (Figs 1 and 2). 1,2,45

Although more expensive, MRI is not operator-dependent. It also demonstrates a more global picture. Fluid-sensitive studies are used in the axial and sagittal planes. We use the following protocol at our centre: proton-density axial and sagittal, T2 fatsaturation axial and sagittal and proton-density fat-saturation sequences. 


\section{ORIGINAL ARTICLE}

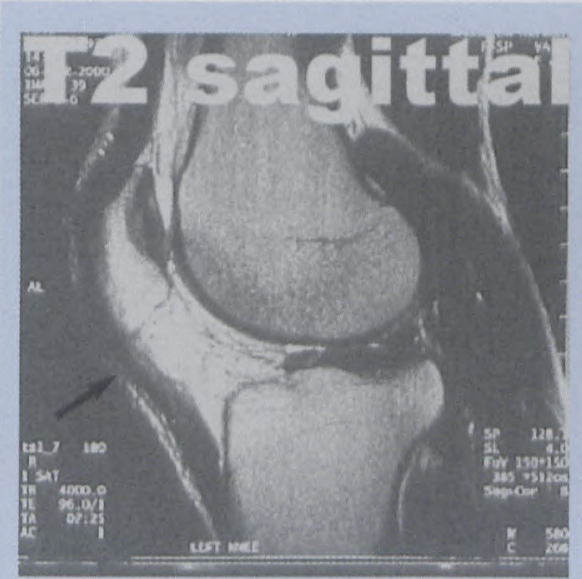

Fig. 4. Sagittal T2 images show similar hyperintense changes to the T1 study of the infrapatellar tendon.

Three millimetre thick sections with no gap, $12 \mathrm{~cm}$ field of view, and $512 \mathrm{x}$ 512 matrix with a standard knee coil completes the protocol. The abnormal areas of tendinosis appear as 'bright' or hyperintense areas in the tendon substance. Associated stress response or stress fracture is demonstrated in the patella. Oedema in the infrapatellar fat pad is well demonstrated on the fat-saturation, fluid-sensitive studies.

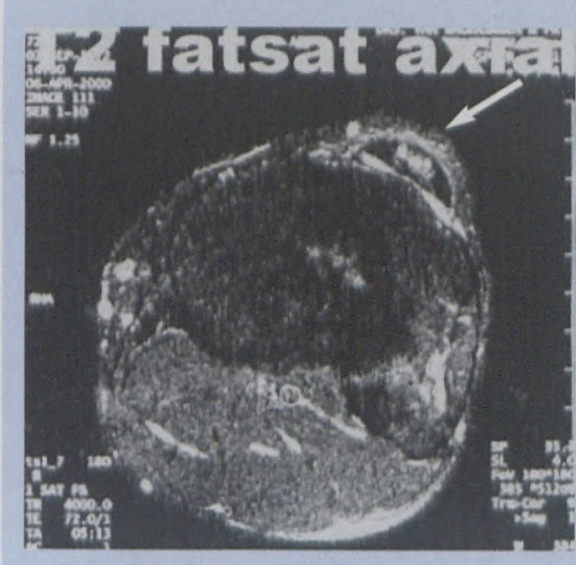

Fig. 5. Transverse T2 fat-saturation study shows marked hyperintense changes in the infrapatellar tendon.

Care must be taken not to confuse the 'magic angle' artefact with tendon pathology. This artefact is seen where the tendon courses at 55 degrees to the coil (Figs 3 - 5). 4,6,7

The pathology of patellar tendinopathy is based on examination of surgical specimens. At surgery the normally white, glistening and firm tendon appears dull, brownish and soft. Microscopically the involved areas show loss of collagen continuity and an increase in ground substance, vascularity and cellularity. The absence of inflammatory cells is stressed, thus the term tendinosis or tendinopathy and not tendonitis. ${ }^{1,2}$

At surgery, either via arthroscopy or longitudinal skin incision, the abnormal tissue is debrided. ${ }^{1}$

\section{References}

1. Khan K, Bonar F, Desmond PM, Cook J,, Young DA, Visentini PJ. Patellar tendinosis: Findings at histopathologic examination, US and MRI. Radiology 1996; 200: 821-827.

2. Anderson IF, Read J, Steinweg J. Atlas of Imaging in Sports Medicine. Sydney: McGraw-Hill, 1998: 216-217.

3. Berquist $\mathrm{TH}$. Imaging of Sports Injuries. Maryland: Aspen Publishers, 1992:149.

4. Bergman AG, Fredericson M. MRI of stress reactions, muscle injuries and other overuse injuries in runners. MRI Clinics of North America 1999: 7:65.

5. Ptasznik R. Musculoskelatal ultrasound. Radiol Clin North Am 1999; 37: 799-803.

6. Stoller D. MRI in Orthopaedics and Sports Medicine. Philadelphia: Lippincott-Raven, 1997: 375-376.

7. Resnick D, Kang H. Internal Derangements of Joints. Emphassis on MR Imaging. Pennsylvania: WB Saunders, 1997: 662-665. 